\title{
Moving Forward in Organizing Acute Pediatric Stroke Care
}

Keywords: Stroke, pediatrics, Neurological practice, thrombolysis

doi:10.1017/cjn.2021.47

Can J Neurol Sci. 2021; 48: 750-751

Arterial ischemic stroke is a pediatric emergency due to risk for permanent life-long disability, high stroke recurrence, and even death. ${ }^{1,2}$ Prompt management interventions have shown to improve stroke outcomes. ${ }^{2,3}$ In adults, recommended acute management interventions include emergent use of systemic tissue plasminogen activator (tPA), neuroprotection, endovascular recanalization in selected cases, and stroke prevention utilizing care pathways, teams, and units. ${ }^{3}$ As timely and accurate diagnosis is a prerequisite for optimal delivery of acute stroke interventions, an exemplary timedependent approach has been adopted by adult neurologists. Guided by adult data, pediatric stroke community has undertaken huge steps to optimize timely diagnosis for delivery of stroke interventions proven to be effective in adults. However, this has been an uneven road as childhood stroke carries unique challenges (low numbers, frequent stroke mimics, varying risk factors, inadequate pediatric evidence and infrastructure resources, delicate ethical and safety issues). ${ }^{2}$ Acknowledging this, pediatric stroke experts have been promoting and leveraging for better organization of acute stroke care at pediatric institutions. ${ }^{2,4}$

The current consensus recommendations for childhood stroke include timely diagnosis, use of neuroprotective and stroke prevention strategies, risk factor evaluation, and age-appropriate rehabilitation. ${ }^{2,5,6}$ Institutional organization of pediatric stroke management utilizing acute protocols and expert multidisciplinary teams has been stressed by all, as these are known to improve management delays and overall caregiver satisfaction. Systemic thrombolysis with tPA and endovascular thrombectomy are areas of knowledge gap and controversy. ${ }^{2}$ In light of recent dedicated pediatric data, current guidelines support these hyperacute interventions in children, provided certain conditions are met. ${ }^{2,6}$ Specifically, the recent American Heart Association guidelines state that these interventions may be considered in select hyperacute pediatric stroke cases (persisting deficits, confirmed intracranial artery occlusion, and older and larger children), provided management is undertaken by experts in pediatric stroke and neurovascular interventional procedures and in hospitals equipped with stroke management pathways. ${ }^{2}$

Over the last decade, many pediatric hospitals have established active stroke protocols and alerts. ${ }^{7,8}$ However, considerable differences have been observed. ${ }^{7}$ The article by Gladkikh et al. in this issue of the Journal provides important insight into these variations and the degree of preparedness for pediatric stroke in Canada. ${ }^{9}$ This study is a qualitative cross-sectional survey of pediatric neurologists on acute stroke protocols at all 16 pediatric hospitals in Canada.
The article by Gladkikh et al. highlights several points. The study confirms that most pediatric hospitals have active stroke pathways, while others are either developing or have a mechanism to manage stroke acutely. While several elements are shared, few notable differences were observed across protocols. The consensus for initial laboratory evaluation and neuroprotection for childhood stroke provides assurance that these follow current guidelines. ${ }^{2}$ Although magnetic resonance imaging (MRI) brain is preferred neuroimaging modality in children with stroke symptoms, ${ }^{2}$ variability in initial neuroimaging choice noted in this study (CT vs. MRI) reflects a real-world scenario, as availability of these resources, particularly after hours, vary across pediatric institutions.

For systemic tPA administration, main indication (stroke onset $<4.5$ hours), contraindications, and exclusions across protocols mirror adult stroke pathways, whereas age-dependent indications appear to follow the famous multicenter Thrombolysis in Pediatric Stroke Study (TIPS), which is not a surprising finding. Until pediatric clinical trials data are available, safe off-label use of tPA for childhood stroke will continue to be guided by adult data and TIPS experience. TIPS was a phase 1 dose finding study of intravenous tPA (dose tiers $0.75,0.9$, and $1.0 \mathrm{mg} / \mathrm{kg} / \mathrm{d}$ ) in children aged $2-18$ years, $<4.5$ hours stroke symptoms onset, $>4-6$ Pediatric National Institute of Health Stroke Scale (PedNIHSS), and confirmation of ischemic stroke with intracranial arterial occlusion. ${ }^{10}$ In the article by Gladkikh and colleagues, although most elements follow TIPS design, age-related inclusion criteria across protocols varied. Although TIPS considered various age-dependent factors (anatomical, homeostatic, and pharmacokinetic), ${ }^{10}$ these factors remain an area of controversy due to lack of dedicated pediatric clinical trials data. Inconsistent use of Alberta Stroke Program Early CT Score (ASPECTS), ${ }^{11}$ a scale routinely used in adults, is explained by the relative lack of experience and comfort with this measure among pediatricians. Encouragingly, all seven pediatric institutions offer endovascular recanalization procedures for acute stroke with demonstrable intracranial arterial occlusion. A preference for mechanical thrombectomy in this study is due to the emerging good quality pediatric data showing its safety and efficacy in children, comparable to adult data. ${ }^{12}$ Two protocols with no specified maximum time limit for mechanical thrombectomy are concerning, but it is likely that they have a 24-hour upper limit as in adults. Of note, two centers continue to offer intra-arterial tPA, although it may increase risk of hemorrhage in children ${ }^{13}$ and is no longer favored by adult stroke guidelines. ${ }^{3}$ This practice may reflect a lag in updating protocols. 
The study has important limitations. Data on the initial triaging process leading to stroke protocol/code activation at each center are lacking. These initial steps are key elements in reducing time lag from symptom onset to stroke intervention. In children, the median delay to stroke diagnosis averages 24 hours. ${ }^{14,15}$ Contrary to adults, children with stroke are brought to medical attention faster (median 1.7 hours), whereas in-hospital delays are longer (median 12.9 hours). ${ }^{14}$ Implementation of stroke protocols in pediatric hospitals has been shown to reduce these delays. ${ }^{15}$ Standard stroke screening tools, shown to effectively reduce diagnostic delays in adults, ${ }^{16}$ are infrequently applied and studied in children. ${ }^{17}$ Details regarding involvement of other disciplines in childhood stroke management are not provided. Notably, details of initial stroke prevention treatment (an area known to lack consensus) are missing, which would have been helpful in assessing practice trends in Canada. As demonstrated by TIPS, resources required to maintain an active stroke protocol at pediatric hospitals are more taxing than adults. Although beyond the scope of this study, a current understanding of these infrastructure and personnel resources will assist streamlining pediatric stroke care, particularly for institutions that currently do not have stroke pathways. Finally, as stated by authors, the results of this study may not be generalizable as treatment practices and systems of care undoubtedly vary across countries.

Acute stroke protocols and pathways are imperative for timely management of stroke. Although pediatric stroke numbers are low, recent data support use of adult-recommended hyperacute stroke interventions in children. Consensus regarding the initial diagnostic evaluation and supportive management of childhood acute ischemic stroke, also noted in this study, are steps toward standard stroke care. Moving forward, better organization and standardization of stroke pathways and systems of care in pediatric hospitals, particularly surrounding hyperacute stroke interventions, are necessary for optimizing the number of children who can potentially receive these interventions.

\section{Disclosures}

The author has no conflict of interest.

\section{Mubeen F. Rafay \\ Department of Pediatric and Child Health, University of Manitoba, Children's Hospital Research, Institute of Manitoba, Winnipeg, Manitoba, Canada}

Correspondence to: Mubeen F. Rafay, Section of Pediatric Neurology, Department of Pediatric and Child Health, University of Manitoba, Children's Hospital Research Institute of Manitoba,
AE 308, 820 Sherbrook Street, Winnipeg, Manitoba R3A 1R9, Canada. Email: mubeen.rafay@utoronto.ca

\section{REFERENCES}

1. DeVeber G, Kirton A, Booth FA, et al. Epidemiology and outcomes of arterial ischemic stroke in children: the Canadian pediatric ischemic stroke registry. Pediatr Neurol. 2017;69:58-70.

2. Ferriero DM, Fullerton HJ, Bernard TJ, et al. Management of stroke in neonates and children: a scientific statement from the American heart association/American stroke association. Stroke. 2019;50: e51-96.

3. Powers WJ, Rabinstein AA, Ackerson T, et al. 2018 Guidelines for the early management of patients with acute ischemic stroke: a guideline for healthcare professionals from the American Heart Association/ American Stroke Association. Stroke. 2018;49:e46-110.

4. Roach ES, Bernard T, DeVeber G. Defining a pediatric stroke center. Pediatr Neurol. 2020;112:11-3.

5. Monagle P, Chan AKC, Goldenberg NA, et al. Antithrombotic therapy in neonates and children: antithrombotic therapy and prevention of thrombosis. Chest. 2012;141:e737S-801S.

6. Medley TL, Miteff C, Andrews I, et al. Australian clinical consensus guideline: the diagnosis and acute management of childhood stroke. Int J Stroke. 2019;14:94-106.

7. Bernard TJ, Rivkin MJ, Scholz K, et al. Emergence of the primary pediatric stroke center: impact of the thrombolysis in pediatric stroke trial. Stroke. 2014;45:2018-23.

8. Bernard TJ, Friedman NR, Stence NV, et al. Preparing for a "pediatric stroke alert". Pediatr Neurol. 2016;56:18-24.

9. Gladkikh M, McMillan HJ, Andrade A, et al. Pediatric hyperacute arterial ischemic stroke pathways at Canadian tertiary care hospitals. Can J Neurol Sci. 2021;48:831-8.

10. Rivkin MJ, DeVeber G, Ichord RN, et al. Thrombolysis in pediatric stroke study. Stroke. 2015;46:880-5.

11. Pexman JH, Barber PA, Hill MD, et al. Use of the Alberta stroke program early CT score (ASPECTS) for assessing CT scans in patients with acute stroke. AJNR Am J Neuroradiol. 2001;22:1534-42.

12 Sporns PB, Strater R, Minnerup J, et al. Feasibility, safety, and outcome of endovascular recanalization in childhood stroe: the Save ChildS study. JAMA Neurol. 2020;77:25-34.

13. Ellis MJ, Amlie-Lefond C, Orbach DB. Endovascular therapy in children with acute ischemic stroke: review and recommendations. Neurology. 2012;79:S158-64.

14. Rafay MF, Pontigon AM, Chiang J, et al. Delay to diagnosis in acute pediatric arterial ischemic stroke. Stroke. 2009;40: 58-64.

15. Shack M, Andrade A, Shah-Basak PP, et al. A pediatric institutional acute stroke protocol improves timely access to stroke treatment. Dev Med Child Neurol. 2017;59:31-7.

16. Zhelev Z, Walker G, Henschke N, Fridhandler J, Yip S. Prehospital stroke scales as screening tools for early identification of stroke and transient ischemic attack. Cochrane Database Syst Rev. 2019;4:CD011427.

17. Yock-Corrales A, Babl FE, Mosley IT, Mackay MT. Can the FAST and ROSIER adult stroke recognition tools be applied to confirmed childhood arterial ischemic stroke? BMC Pediatr. 2011;11:93. 